\title{
Modernes Wundmanagement chronischer Wunden
}

\author{
Modern Wound Management of Chronic Wounds
}

Autor

Institut

\section{J. Dissemond}

Klinik und Poliklinik für Dermatologie, Venerologie und Allergologie, Universitätsklinikum Essen
Bibliografie

Dol $10.1055 / \mathrm{s}-2008-1077581$

Akt Dermatol 2008; 34 :

386-391 @ Georg Thieme

Verlag KG Stuttgart · New York ISSN 0340-2541

Korrespondenzadresse

Priv.-Doz. Dr. med.

Joachim Dissemond

Universitätsklinikum Essen

Klinik und Poliklinik für

Dermatologie, Venerologie

und Allergologie

Hufelandstraße 55

45122 Essen

joachimdissemond@

hotmail.com

\section{Zusammenfassung \\ $\nabla$}

In den westlichen Industrienationen kann eine steigende Zahl von Patienten mit chronischen Wunden beobachtet werden. Die zugrunde liegenden ätiopathogenetisch relevanten Faktoren sind sehr vielfältig. Nach interdisziplinärer Durchführung einer umfassenden Diagnostik, stellt die kausal orientierte Therapie die Basis der Behandlung der Patienten dar. Begleitend kann eine moderne, feuchte und an den Phasen der Wundheilung orientierte Wundtherapie eingeleitet werden. Neben den modernen Verbandstoffen, die in verschiedene Kategorien wie passiv, interaktiv und aktiv eingeteilt werden kön-

\section{Einleitung \\ $\nabla$}

Es wird geschätzt, dass in den westlichen Industrienationen etwa 1-2\% der Bevölkerung unter einer chronischen Wunde leiden. Die Prävalenz steigt jedoch erheblich mit zunehmendem Lebensalter an, sodass sie nach dem 80 . Lebensjahr etwa $4-5 \%$ betragen soll $[6,21]$. Eine zuverlässige Erhebung dieser Daten erfolgt in Deutschland derzeit allerdings nicht. Ein weiteres Problem ergibt sich daraus, dass zudem bislang keine einheitliche Definition einer chronischen Wunde existiert. So wurde beispielsweise vorgeschlagen, eine Wunde dann als chronisch zu bezeichnen, wenn diese mindestens seit 3 Monaten besteht [5].

In Abhängigkeit des untersuchten Patientenkollektivs sollen mindestens $50 \%$ aller chronischen Wunden sich als Ulcus cruris ( Abb. 1), 25\% als Decubitus ( Abb. 2) und 15\% im Rahmen eines Diabetes mellitus an den Füßen ( $\bullet$ Abb. 3) manifestieren [8]. Darüber hinaus existieren zahlreiche weitere Faktoren wie beispielsweise eine periphere arterielle Verschlusskrankheit ( $\bullet$ Abb.4), Vasculitis ( $\bullet$ Abb.5), arterieller Hypertonus, nen, sind zunehmend innovative weitere Therapieoptionen verfügbar. So können heute beispielsweise Vakuumtherapien, Ultraschallbehandlungen, Hydrotherapien oder die Biochirurgie eingesetzt werden. Hoffnungsvolle zukünftige Therapieverfahren, für die bereits erste klinische Erfolge beschrieben wurden, könnten neben dem weiter differenzierten und individuell adaptierten Einsatz von Wachstumsfaktoren auch beispielsweise TNF- $\alpha$ Inhibitoren, Stammzellen oder Knochenmarkzellen sein. Somit kann der Einsatz einer modernen Wundtherapie, eingebunden in ein kausal ansetzendes Therapiekonzept, dazu beitragen, die Abheilung zuvor chronischer Wundverläufe zu gewährleisten.

Malnutrition oder Medikamente, die eine chronische Wunde verursachen können oder deren Abheilung zumindest wesentlich behindern (๑ Tab. 1) [6]. Da die Ursachen für die Entstehung chronischer Wunde sehr vielfältig sein können, ist meist ein interdisziplinärer diagnostischer und therapeutischer Ansatz sinnvoll [10].

\section{Diagnostik \\ $\nabla$}

Der erste Schritt der Behandlung der Patienten mit chronischen Wunden muss immer die Objektivierung der kausal relevanten Faktoren darstellen. So kann oft schon durch die Anamnese und eine klinische Inspektion eine Verdachtsdiagnose geäußert werden. Anschließend werden entsprechend der klinischen Befunde u. a. weiterführende apparative und serologische Untersuchungen notwendig sein ( Tab. 2) [10]. Eingebunden in ein Gesamtkonzept kann schließlich eine moderne feuchte Wundtherapie die Abheilung einer chronischen Wunde fördern. 


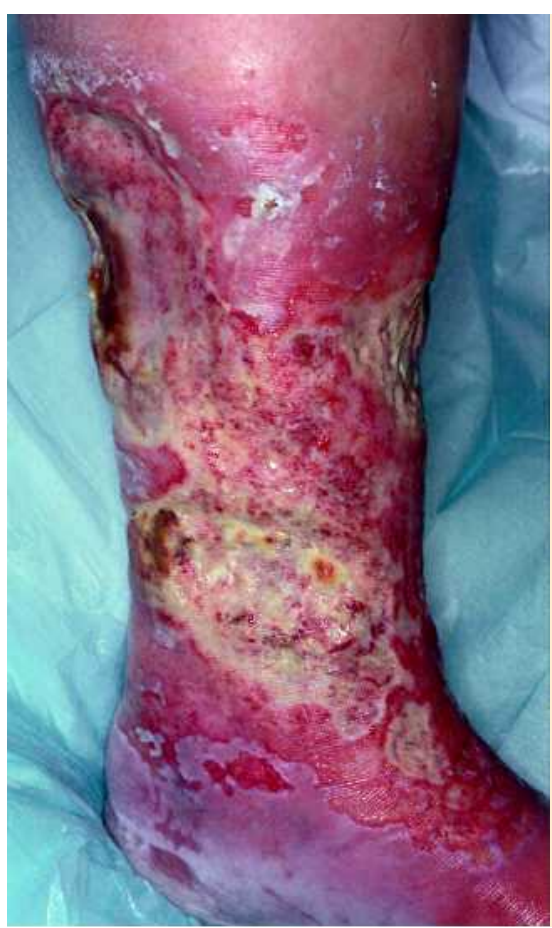

Abb. 1 Ulcus cruris venosum.

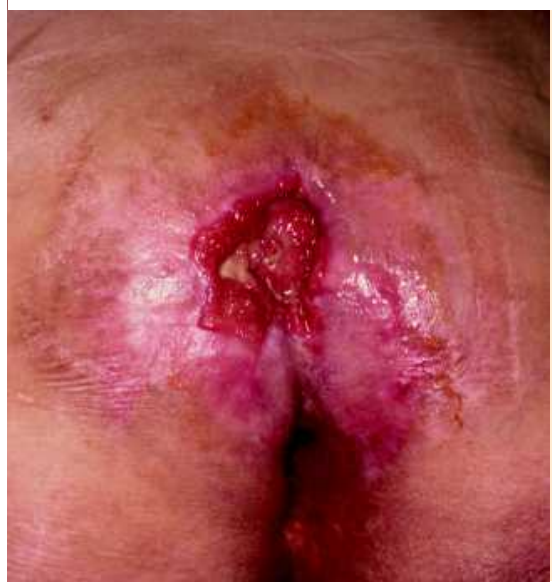

Abb. 2 Sacral lokalisierter Decubitus.

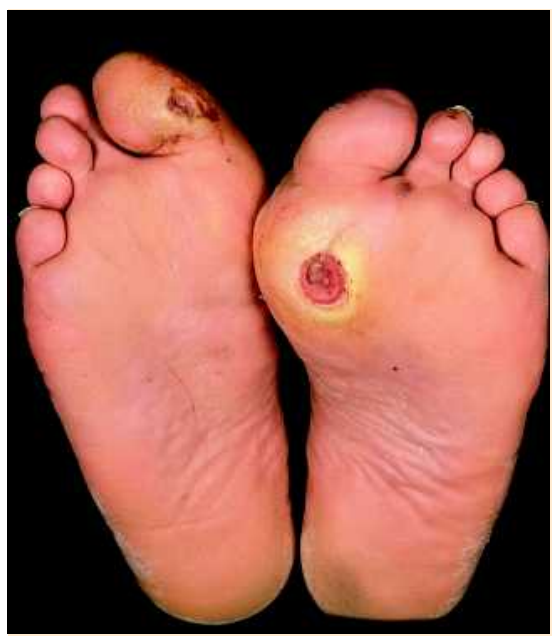

Abb. 3 Diabetisches trophen Ulzerationen Digitus I.

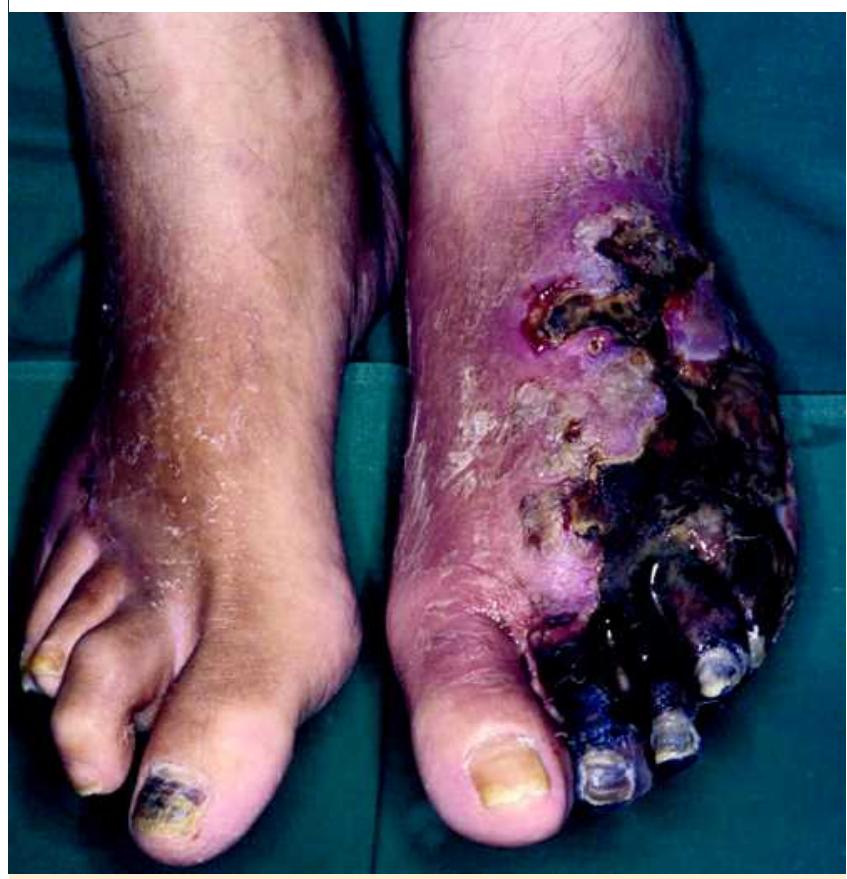

Abb. 4 Patient mit Ulzerationen bei fortgeschrittener pAVK.

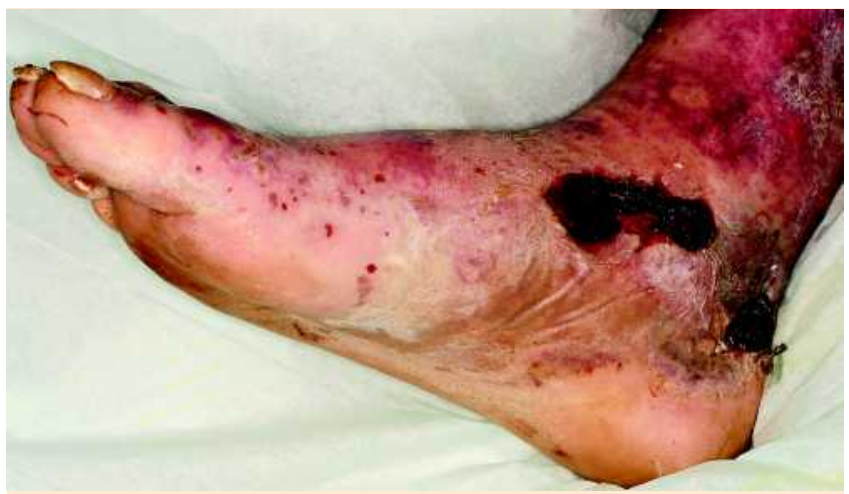

Abb. 5 Multiple Ulzerationen beider distaler Extremitäten im Rahmen einer leukozytoklistischen Vasculitis.

\section{Moderne Wundtherapie} Fußsyndrom mit neuroplantar und jeweils am
Als Wegbereiter der heute modernen Wundtherapie wird meist der Brite George D. Winter genannt, der bereits 1962 mit seinen grundlegenden Untersuchungen in einem Schweinemodel zeigen konnte, dass artefizielle Wunden durch Anwendung eines okklusiven Folienverbandes beschleunigt abheilen. Seit dieser Zeit sind zahlreiche klinische Studien publiziert worden, die den Vorteil der feuchten Wundbehandlung verglichen mit einer trockenen Wundbehandlung auch bei Patienten eindeutig belegen $[13,14]$. Obwohl ein Pflegedienst durchschnittlich etwa 17 Minuten für die Durchführung eines Wundverbandes benötigt, wird geschätzt, dass derzeit lediglich $10-20 \%$ aller Patienten mit einer chronischen Wunde in Deutschland eine moderne feuchte Wundtherapie erhalten [20]. 


\begin{tabular}{|c|c|c|c|}
\hline Genese & Lokalisation & Wundfläche, Randsaum & Umgebung \\
\hline CVI & $\begin{array}{l}\text { Oberhalb oder hinter } \\
\text { Malleolus medialis }\end{array}$ & $\begin{array}{l}\text { Unscharfe Begrenzung, unterminiert, } \\
\text { wenig Nekrosen, Fibrinbeläge, } \\
\text { oft wenig schmerzhaft }\end{array}$ & $\begin{array}{l}\text { Ödem, Pigmentierung, } \\
\text { Sklerose, Ekzem }\end{array}$ \\
\hline PAVK & Akral insb. Zehen & $\begin{array}{l}\text { Scharfe Begrenzung, Nekrosen, } \\
\text { schmerzhaft }\end{array}$ & $\begin{array}{l}\text { Blässe, Haarverlust, } \\
\text { Atrophie }\end{array}$ \\
\hline Diabetes & $\begin{array}{l}\text { Plantar insb. unter } \\
\text { Metatarsale-Köpfchen } \\
\text { oder Zehen }\end{array}$ & $\begin{array}{l}\text { Scharfe Begrenzung, unterminiert } \\
\text { Hyperkeratosen, wenig Nekrosen, } \\
\text { kaum schmerzhaft }\end{array}$ & Hyperkeratosen \\
\hline Vasculitis & $\begin{array}{l}\text { Multifokal, ansonsten } \\
\text { „untypische“Areale }\end{array}$ & $\begin{array}{l}\text { Scharfe Begrenzung, bizarr konfiguriert, } \\
\text { Nekrosen, sehr schmerzhaft }\end{array}$ & Purpura, Erythem \\
\hline
\end{tabular}

Tab. 1 Typische klinische Symptome bei chronischen Wunden der unteren Extremitäten.

\begin{tabular}{|c|c|c|c|}
\hline Diagnostik & Serologie & Testungen & Apparativ \\
\hline Minimum & Blutbild, CRP & $\begin{array}{l}\text { Mikrobiologie, } \\
\text { Pulstastung }\end{array}$ & Doppler, brachio-tibialer Index \\
\hline Standard & $\begin{array}{l}\mathrm{HbA}_{1} \mathrm{c}, \mathrm{BSG}, \mathrm{TPZ}, \mathrm{PTT} \text {, Gesamt- } \\
\text { eiweiß, Differenzialblutbild, } \\
\text { Elektrolyte }\end{array}$ & Epikutan-Testung & Duplex \\
\hline Erweitert & $\begin{array}{l}\text { zirkulierende Immunkomplexe, } \\
\text { Kryoglobuline, Homocystein, AT-III, } \\
\text { PAI-1, APC-Resistenz, Vitamine, } \\
\text { Protein C, Protein S, Paraproteine, } \\
\text { Spurenelemente, ANA, ENA, } \\
\text { ANCA, dsDNA, Antiphospholipid- } \\
\text { Antikörper, Harnstoff, Kreatinin, } \\
\text { Tetanus, Blutfette }\end{array}$ & $\begin{array}{l}\text { Biopsie, Raynaud- } \\
\text { Test, Pathergie-Test }\end{array}$ & $\begin{array}{l}\text { Angiografie, Sauerstoffpar- } \\
\text { tialdruck, Kapillarmikroskopie, } \\
\text { Lymphografie, Röntgen/CT/ } \\
\text { MRT, Phlebografie, Licht- } \\
\text { Reflexions-Rheografie, } \\
\text { Venen-Verschluss-Plethysmo- } \\
\text { grafie, Phlebodynamometrie }\end{array}$ \\
\hline
\end{tabular}

Tab. 2 Diagnostische Verfahren bei chronischen Wunden der unteren Extremitäten.

\section{Wundspïlung \\ $\nabla$}

Im Rahmen eines Verbandwechsels sollte eine Wunde immer auch gereinigt werden. Als Wundspüllösungen sollten heute ausschließlich angewärmte und sterile 0,9\% Kochsalzlösung oder Ringerlösung eingesetzt werden. Der Einsatz von Leitungswasser bleibt weiterhin umstritten [6,11]. Da insbesondere im ambulanten Bereich kaum Alternativen zur Verfügung stehen, ist hier zusätzlich die Verwendung eines vorgeschalteten Sterilfilters zu empfehlen. Bei Wasserduschen kann zusätzlich der mechanische Reinigungseffekt genutzt werden. Die Anwendung von Bädern sollte hingegen strikt gemieden werden. Falls zusätzlich eine Desinfektion notwendig ist, sollten bei Patienten mit chronischen Wunden ausschließlich nicht-zytotoxische Antiseptika mit Octenidin oder Polihexanid verwendet werden [16]. Beide Antiseptika sind auch als Bestandteil von Hydrogelen und einigen Wundauflagen erhältlich, sodass der antiseptische Effekt mit einer modernen Wundtherapie kombiniert werden kann. Bei lokal infizierten Wunden können alternativ auch Wundverbände mit Silber eingesetzt werden.

\section{Debridement \\ $\nabla$}

Als Debridement bezeichnet man die Entfernung von nichtvitalem Gewebe aus einer Wunde. Neben der Entfernung von Nekrosen ist hier auch eine Abtragung von Fibrin, Verbandresten oder Fremdkörpern erforderlich [8]. Das sachgerechte Debridement stellt eine grundlegende Voraussetzung für den Ablauf eines nicht verzögerten Wundheilungsprozesses dar.

\section{Chirurgie}

Das chirurgische Debridement stellt weiterhin die Methode der ersten Wahl dar, um möglichst rasch auch größere Wunden zu versorgen. Vor der operativen Maßnahme ist unbedingt auf eine ausreichende Analgesie zu achten [2]. Neben den meist eingesetzten Instrumenten Skalpell und/oder „scharfer“ Löffel hat sich insbesondere der Einsatz von Ringküretten bewährt (० Abb. 6).

\section{Proteolytische Enzyme}

Proteolytische Enzyme ermöglichen im Rahmen einer enzymatischen Wundreinigung eine schmerzfreie, selektive Nekrolyse ohne Blutungen. Das Auftragen in Form einer Salbe oder eines Hydrogels ist einfach, sicher und schnell durchzuführen. Die in Deutschland derzeit erhältlichen Präparate beinhalten eine Clostridium histolyticum Collagenase oder eine Mischung aus Streptodornase und Streptokinase [8].

\section{Biochirurgie}

Die Wundbehandlung mit steril gezüchteten Fliegenmaden wird auch als Biochirurgie bezeichnet. Es werden ca. 5-10 Maden/ $\mathrm{cm}^{2}$ Wundoberfläche für 3-4 Tage auf die Wunden aufgebracht (๑ Abb.7). Alternativ zu den ursprünglich ausschließlich genutzten sogenannten Freiläufern können die Fliegenmaden auch eingearbeitet in einen Verband („Biobag“) erworben werden. Die Fliegenmaden sind obligate Nekrophagen und können so im Rahmen ihrer extrakorporalen Verdauung eine äußerst selektive Nekrolyse durchführen, die von den meisten Patienten als wenig schmerzhaft empfunden wird. Weiterhin eignet sich die Biochirurgie auch für die Behandlung einer lokalen Infektion, insbesondere auch bei Nachweis von grampositiven „Problemkeimen“ wie beispielsweise Methicillin resistenten Staphylococcus aureus (MRSA) [9]. 


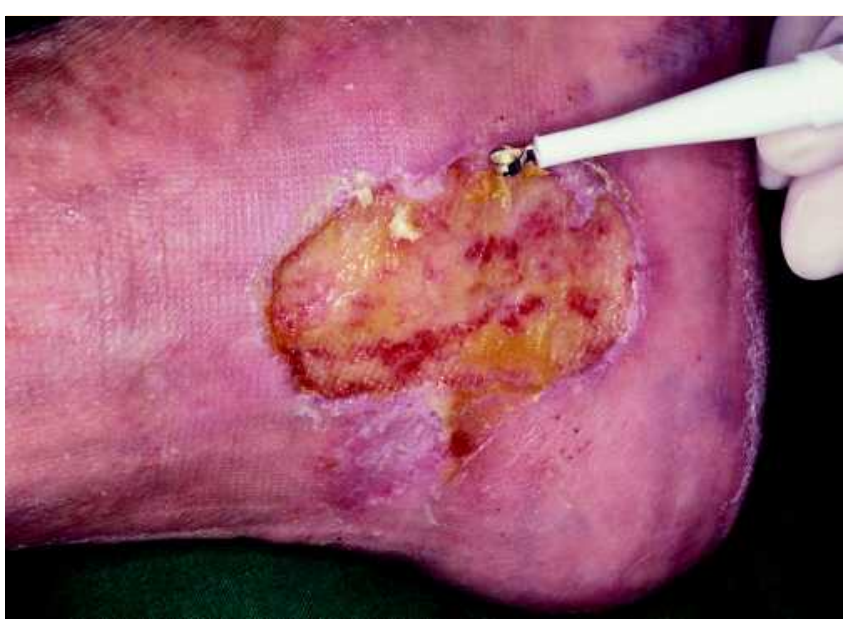

Abb. 6 Chirurgisches Debridement mittels Ringkürette.

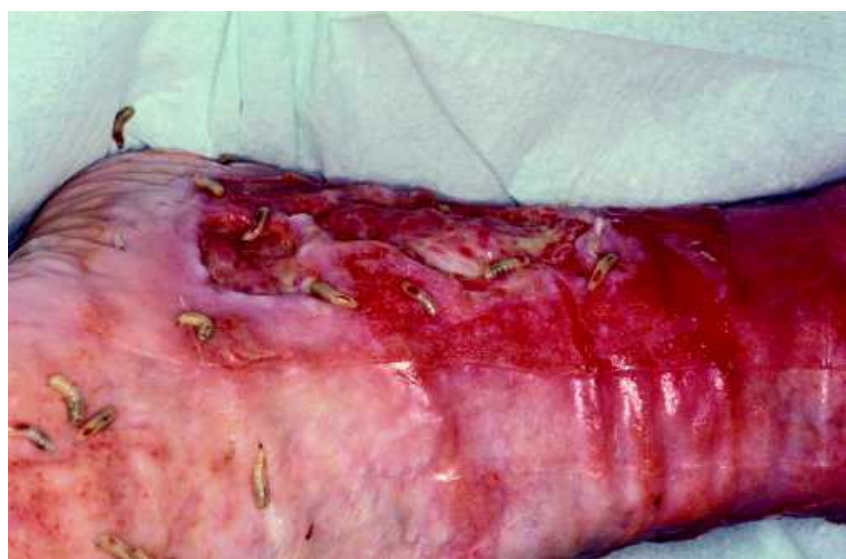

Abb. 7 Biochirurgen auf einem Ulcus cruris, nachdem der Schutzverband nach 3 Tagen entfernt wurde.

\section{Hydrotherapie}

Unter dem Begriff der Hydrotherapie versteht man verschiedene Methoden, bei denen Flüssigkeit für die Säuberung der Wunden Verwendung findet. Bei der als Hochdruck-Irrigation bezeichneten Methode wird die Spülflüssigkeit durch Druck von bis zu 800 bar auf Geschwindigkeiten von bis zu $1000 \mathrm{~km} / \mathrm{h}$ beschleunigt. Zusätzlich zu der physikalischen Nekrosektomie wird eine effektive Elimination von Mikroorganismen durch den Spüleffekt beschrieben [8].

\section{Ultraschall}

Niederfrequenter (Leistungs-)Ultraschall wird neben der propagierten Förderung der Granulation auch für die Abtragung von avitalem Gewebe in Wunden eingesetzt. Insbesondere durch den in den letzten Jahren weiterentwickelten sogenannten Ultraschall-Dissektor kann die Intensität des sehr effektiven physikalischen Debridements individuell adaptiert erfolgen (O Abb. 8) $[1,8]$.

\section{Wundauflagen}

Moderne Wundtherapeutika können in verschiedene Kategorien eingeteilt werden. So kann man beispielsweise versuchen, die zunehmende Anzahl von Wundauflagen in „inaktiv/passiv“, „interaktiv“ und „aktiv“ zu unterteilen. Nach einer Einteilung von

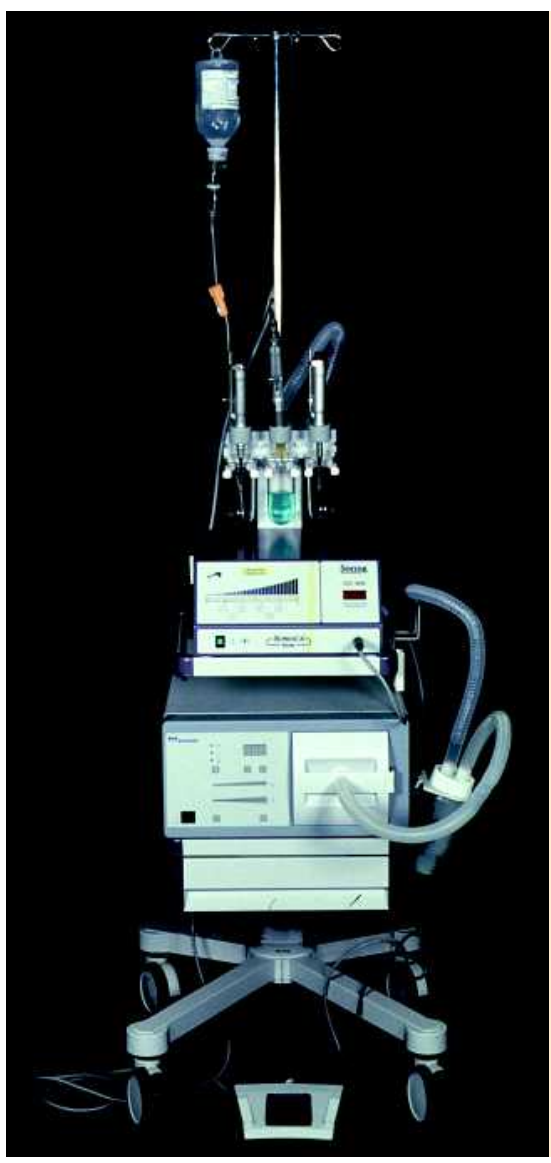

Abb. 8 Ultraschalldissektor.

\section{Aktivkohle}

Die Aktivkohleverbände bestehen aus einem Faserverbund von zuvor verkohlten Zelluloseprodukten, die Geruch absorbieren, bakterizid wirken und Endotoxine aufnehmen. Aktivkohleverbände können insbesondere bei bakteriell kontaminierten und stark sezernierenden Wunden verwendet werden.

Alginat

Wundauflagen aus Alginaten werden aus Fasern von Rot- oder Braunalgen hergestellt und wandeln sich nach Kontakt mit Blut oder Wundsekret unter Quellung in ein hydrophiles Gel um, in das Bakterien und Detritus eingeschlossen wird. Alginate können auch in tiefe bzw. zerklüftete Wunden eingebracht werden und eignen sich für die Behandlung von stark sezernierenden, 
verschmutzten und/oder bakteriell kontaminierten Wunden. Aufgrund der hämostyptischen Wirkung sind Alginate auch für die Behandlung von blutenden Wunden geeignet.

\section{Hydrofaser}

Die Flüssigkeitsaufnahme erfolgt bei Hydrofasern bis zu dem 40-Fachem des Eigengewichtes innerhalb weniger Minuten und ausschließlich vertikal, sodass Mazerationen im Wundrandbereich verhindert werden. Nach der Sekretabsorption wandeln sich die Fasern in transparentes Gel um. Hydrofasern werden insbesondere bei stark sezernierenden Wunden für die Förderung der Granulation eingesetzt.

\section{Hydrogel}

Hydrogele, die auch für ein schmerzloses autolytisches Debridement eingesetzt werden, können sowohl Feuchtigkeit an die Wunde abgeben als auch überschüssiges Wundexsudat aufnehmen. Aufgrund dieser Eigenschaft können sie auch mit vielen anderen Produkten insbesondere bei sehr trockenen Wunden kombiniert eingesetzt werden.

\section{Hydrokolloid}

Hydrokolloidverbände bestehen aus einem Polyurethanfilm oder einem Schaumstoff, auf dem eine selbstklebende, stark quellende Masse aufgebracht ist. Unter Aufnahme von Wundexsudat verflüssigt sich die Hydrokolloidmasse und bildet ein visköses Gel. Durch ihre hydroaktiven Eigenschaften sind die Hydrokolloide in der Lage, fibrinöse Beläge aufzuweichen und abzulösen. Hydrokolloidverbände erzeugen ein feuchtes, okklusives Wundmilieu und werden insbesondere zur Förderung der Granulation verwendet. Der Verband sollte den Wundrand um ca. $2-3 \mathrm{~cm}$ überlappen, um einerseits eine ausreichende Haftung zu gewährleisten und andererseits die umgebende gesunde Haut nicht zu mazerieren.

\section{Kollagen}

Kollagenprodukte werden als Flies, Pulver oder Schwamm angeboten und sollen als temporärer Dermisersatz insbesondere für die Aktivierung bei stagnierender Wundheilung eingesetzt werden. Aufgrund der hämostyptischen Eigenschaften finden sie auch beispielsweise nach einem chirurgischen Debridement Einsatz.

\section{Schaumstoff}

Moderne Schaumstoffkompressen bestehen aus geschlossenporigem Polyurethanschaum. Die Oberflächen sind beispielsweise mit Silikon beschichtet oder thermisch geglättet, um einen atraumatischen Verbandwechsel zu ermöglichen. Schaumstoffkompressen können nach einem Debridement in jeder Phase der Wundheilung Anwendung finden. Hingegen werden die früher oft für die Wundgrundkonditionierung eingesetzten offenporigen Schaumstoffkompressen, in die Granulationsgewebe einwächst, aufgrund der schmerzhaften Verbandwechsel heute als obsolet gesehen.

\section{Vakuumtherapie \\ $\nabla$}

Seit etwa einem Jahrzehnt werden die modernen Systeme der Vakuumtherapie weltweit zunehmend für die Behandlung chronischer Wunden eingesetzt. Das System der Vakuumtherapie besteht aus einem sterilen auswechselbaren Schwamm und ei-

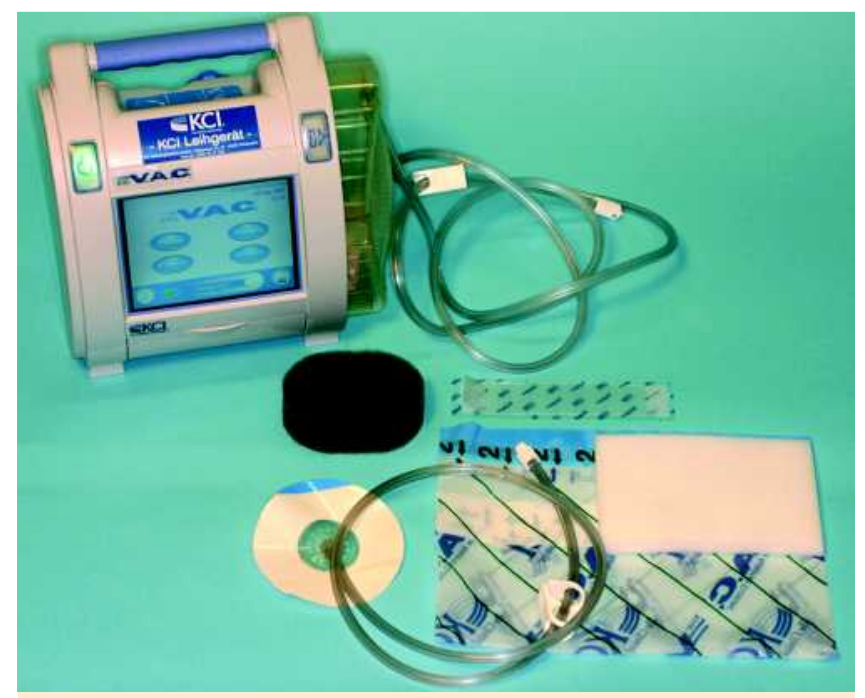

Abb. 9 Utensilien, die für die Durchführung einer Vakuumtherapie benötigt werden.

nem nicht kollabierbaren Schlauchsystem mit einer Therapieeinheit, die einen Unterdruck erzeugt ( $\bullet$ Abb. 9). Der Schwamm wird entsprechend der Größe der zu behandelnden Wunde ausgewählt und ggf. so zurechtgeschnitten, dass er möglichst nicht über den Wundrand reicht. Die Wunde wird dann mit einer transparenten Polyurethanfolie abgedeckt und das Schlauchsystem auf bzw. in dem Schwamm befestigt. Die elektronische Pumpe kann dann einen individuell modulierbaren Sog über das Drainagesystem aufbauen. Die Behandlung chronischer Wunden wird meist mit einem Sog von 125 mm Hg und Verbandwechselintervallen von 2-4 Tagen durchgeführt. Durch den Sog wird die Wunde von Mikroorganismen und Wundsekret mechanisch gereinigt. Zudem stellt das geschlossene Verbandsystem eine physikalische Barriere gegenüber exogenen Einflüssen und insbesondere gegenüber Superinfektionen dar. Der negative zentripedale Druck zieht die Wundränder zentral zusammen, wobei die physiologische Wundkontraktion unterstützt, das interstitielle Ödem reduziert und die Mikrozirkulation angeregt wird. Diskutiert wird darüber hinaus eine direkte Steigerung der Blutflussgeschwindigkeit sowie eine verbesserte Mikrozirkulation im Rahmen der Verminderung des Wundödems $[7,23]$. Der Einsatzbereich der Vakuumtherapie breitet sich weiter zunehmend aus. So wird beispielsweise durch den postoperativen Einsatz der Vakuumtherapie eine verbesserte Einheilrate bei Spalthauttransplantaten beschrieben [15].

\section{Andere physikalische Therapieverfahren $\nabla$}

Neben der Vakuumtherapie existieren zunehmend physikalische Wundbehandlungsmethoden, die meist nach einem Debridement für die Förderung einer stagnierenden Wundheilung eingesetzt werden sollen. So sollen an dieser Stelle lediglich exemplarisch die Elektrostimulation, Laser- und UV-Behandlung, wassergefilterte Infrarot-A-Wärmestrahlung, hyperbare Sauerstofftherapie, extrakorporale Stoßwellentherapie oder Hyperthermieverfahren genannt werden, deren Wirksamkeit in klinischen Studien meist noch unzureichend belegt wurde $[6,8,17]$. 


\section{Hautersatzverfahren (,tissue-engineering ${ }^{6)}$ $\nabla$}

Der Goldstandard der Reepithelisierung chronischer Wunden ist nach ausreichender Wundgrundkonditionierung die autologe Spalthauttransplantation, meist in Form einer Mesh-graft. Moderne Alternativen stellen Hautersatzverfahren dar. So können beispielsweise auch autologe Keratinozyten aus Hautbiopsien oder Haarwurzeln des Patienten gewonnen werden, die in vitro gezüchtet und anschließend mittels Fibrinkleber oder auf Silikonscheiben auf die Wunde appliziert werden. Zudem können auch dermale Hautersatztransplantate aus humanen oder tierischen Ressourcen gewonnen werden, die dann ggf. gemeinsam mit Keratinozyten aufgetragen werden [6].

\section{Perspektiven \\ $\nabla$}

Für den zukünftigen Einsatz bei symptomatischen Wundbehandlungsstrategien werden fortlaufend sehr interessante in vitro-Daten vorgestellt. Bedauerlicherweise werden nur wenige dieser therapeutischen Ansätze auch in klinischen Studien weiter verfolgt. Sehr interessante Therapien basieren seit mehreren Jahren bereits auf der Verwendung von verschiedenen Wachstumsfaktoren wie beispielsweise EGF, TGF, VEGF, G(M)-CSF und anderen, von denen bislang ausschließlich PDGF als Wundprodukt erhältlich ist $[3,6]$. Als weitere sehr interessante Therapieansätze, von denen in Zukunft noch hoffnungsvolle Resultate zu erwarten sind, können beispielsweise TNF- $\alpha$ Inhibitoren [22], Stammzellen [24] oder autologe Knochenmarkzellen [18] in der Wundbehandlung genannt werden.

\section{Fazit \\ $\nabla$}

Es ist sicherlich unbestritten, dass eine feuchte und an den Phasen der Wundheilung orientierte Behandlung unter Verwendung moderner Wundauflagen mit dazu beitragen kann, ein optimales Wundmilieu zu erzeugen, und somit die Heilung chronischer Wunden fördert. Jedoch ist auf der Basis einer umfassenden Diagnostik die kausal ansetzende Therapie der zugrunde liegenden Erkrankung(en) immer die wesentliche Voraussetzung, um auch die dauerhafte Abheilung chronischer Wunden der Patienten gewährleisten zu können.

\section{Abstract}

\section{Modern Wound Management of Chronic Wounds $\nabla$}

In Western developed countries a growing incidence of patients with chronic wounds has been described. The underlying etiopathologically relevant causes are manifold and thus interdisciplinary diagnostics and causal therapy are the basis for a good and successful wound treatment. Later a modern, moisten, and phase adapted wound therapy should be initiated as a part of a complex therapeutic concept.

Beside modern wound-dressings which could be divided in for example passive, interactive and active dressings there exists an increasing amount of innovative therapeutic options. For instance negative pressure therapies, treatments with ultrasound, hydrotherapies or biosurgery could be used. Moreover beside the well known growth factors there are some hopeful procedures with first clinical results for future wound healing strategies available like TNF- $\alpha$ inhibitors, stem cells or bone marrow derived cells.

Therefore the employment of a modern wound therapy together with causally orientated treatment of the underlying causes is helpful to ensure a long-term complete healing of chronic wounds.

\section{Literatur}

1 Al-Kurdi D, Bell-Syer SE, Flemming $K$. Therapeutic ultrasound for venous leg ulcers. Cochrane Database Syst Rev 2008; 23: CD001180

2 Briggs M, Nelson EA. Topical agents or dressings for pain in venous leg ulcers. Cochrane Database Sys Rev 2003; 1: CD001177

3 Cianfarani F, Tommasi R, Failla CM et al. Granulocyte/macrophage colony-stimulating factor treatment of human chronic ulcers promotes angiogenesis associated with de novo vascular endothelial growth factor transcription in the ulcer bed. Br J Dermatol 2006; 154: 34 - 341

4 Dissemond J. Moderne Wundauflagen für die Therapie chronischer Wunden. Hautarzt 2006; 10: 881 - 887

5 Dissemond J. Wann ist eine Wunde chronisch? Hautarzt 2006; 57: 55

6 Dissemond J. Ulcus cruris - Grundlagen, Diagnostik und Therapie. 2. Auflage. Bremen, London, Boston: UNI-MED Verlag, 2007

7 Dissemond J. Vakuumtherapie chronischer Wunden in der Dermatologie. Hautarzt, in press

8 Dissemond J, Goos M. Optionen des Debridements in der Therapie chronischer Wunden. J Dtsch Dermatol Ges 2004; 9: 743-751

9 Dissemond J, Koppermann M, Esser $S$ et al. Therapie eines Methicillinresistenten Staphylokokkus aureus (MRSA) im Rahmen der Behandlung eines chronischen Ulcus mittels Biochirurgie. Hautarzt 2002; 53: $608-612$

10 Dissemond J, Körber A, Jansen T, Grabbe S. Aktuelle Diagnostik des Ulcus cruris. Dtsch Med Wochenschr 2005; 130: 1263-1266

11 Fernandez R, Griffiths $R$. Water for wound cleansing. Cochrane Database Syst Rev 2008; 23: CD003861

12 Horn T. Lokale Wundauflagen: Übersicht und Klassifikation. Chir Gastroenterol 2006; 22: 147-154

13 Jones J. Winter's concept of moist wound healing: a review of the evidence and impact on clinical practice. J Wound Care 2005; 14: 273 276

14 Jones KR, Fennie K, Lenihan A. Evidence-based management of chronic wounds. Adv Skin Wound Care 2007; 20: 591 -600

15 Körber A, Franckson T, Lehnen M, Grabbe S, Dissemond J. Vacuum assisted closure device improves take of mesh-grafts in chronic leg ulcer patients. Dermatology 2008; 216: 250-256

16 Kramer A, Daeschlein G, Kammerlander G et al. Konsensusempfehlung zur Auswahl von Wirkstoffen für die Wundantiseptik. ZfW 2004; 3: $110-120$

17 Kranke P, Bennett M, Roeckl-Wiedmann I, Debus S. Hyperbaric oxygen therapy for chronic wounds. Cochrane Database Syst Rev 2004; 2 : CD004123

18 Liu Y, Dulchavsky DS, Gao X et al. Wound repair by bone marrow stromal cells through growth factor production. J Surg Res 2006; 136: $336-341$

19 Nelson EA, Bradley MD. Dressings and topical agents for arterial leg ulcers. Cochrane Database Syst Rev 2003; 1: CD001836

20 Panfil EM, Mayer H, Junge W et al. Die Wundversorgung von Menschen mit chronischen Wunden in der ambulanten Pflege - Pilotstudie. Pflege 2002; 15: 169-176

21 Pirk 0. Chronische Wunden: Viel Geld für nichts? Dtsch Ärzteblatt 2000; 97: 2992-2994

22 Streit M, Beleznay Z, Braathen LR. Topical application of the tumour necrosis factor-alpha antibody infliximab improves healing of chronic wounds. Int Wound J 2006; 3: 171 - 179

23 Ubbink DT, Westerbos SJ, Evans D, Land L, Vermeulen H. Topical negative pressure for treating chronic wounds. Cochrane Database Syst Rev 2008; 16: CD001898

24 Zhang $C P$, Fu XB. Therapeutic potential of stem cells in skin repair and regeneration. Chin J Traumatol 2008; 11: 209-221 